\title{
Epidural analgesia for labor: Current techniques
}

\author{
This article was published in the following Dove Press journal: \\ Local and Regional Anesthesia \\ 7 December 2010 \\ Number of times this article has been viewed
}

\section{Marcos Silva \\ Stephen H Halpern \\ Department of Anesthesia, Sunnybrook Health Sciences Centre and University of Toronto, Toronto, Canada}

\begin{abstract}
Epidural analgesia is an extremely effective and popular treatment for labor pain. In this review, we trace the history of the use of epidural analgesia and its refinements. We then outline the goals of treatment and methods used to attain those goals. The use of low concentrations of local anesthetics, combined with lipid-soluble opioids, does not impede the progress of labor or depress the newborn. The incidence of side effects is low. Maintenance of analgesia that allows patient control enhances patient satisfaction.
\end{abstract}

Keywords: analgesia, labor, epidural, spinal, local anesthesia, childbirth

\section{Introduction}

Epidural analgesia is an extremely effective and popular treatment for labor pain. In Canada, the epidural rate varies between the provinces from $30 \%$ to $69 \%$. $^{1}$ The use of epidural analgesia in the US has tripled between 1981 and 2001, with $60 \%$ of women using this technique in large hospitals. ${ }^{2}$ In this review, we will outline a brief history of the use of epidural analgesia and examine the current techniques of initiation and maintenance of pain relief. We will also discuss the main complications and contraindications for this method of analgesia.

In this review, epidural analgesia refers to local anesthetics and adjuvants injected into the epidural space. Spinal anesthesia refers to local anesthetic, with or without adjuvants, injected into the subarachnoid space. Combined spinal-epidural analgesia includes analgesia initiated with an intrathecal injection and placement of an epidural catheter to provide a route for additional drug. Neuraxial analgesia includes spinal, epidural, and combined spinal-epidural analgesia.

\section{History}

An outline of the history of neuraxial analgesia is shown in Figure 1. The introduction of neuraxial analgesia into obstetric practice took place at the end of the 19th century, one year after August Bier, a German surgeon, described six lower extremity operations rendered painless by means of "cocainization of the spinal cord". Oskar Kreis, a Swiss obstetrician, described total anesthesia of the lower body in six laboring parturients after subarachnoid injection of cocaine. He injected $0.01 \mathrm{~g}$ of cocaine intrathecally at the L4-5 interspace and observed complete pain relief within 5-10 minutes. Like Bier, Kreis observed no serious complications, but severe vomiting and headache occurred frequently. Postdural puncture headache would prove to be one of the main limitations associated with subarachnoid block for labor analgesia.
Correspondence: Stephen Halpern Obstetric Anesthesia, Sunnybrook Health Sciences Centre, 2075 Bayview Ave, Room M5 3I8-E, Toronto, Ontario, Canada M4N 3M5

$\mathrm{Tel}+\mathrm{I} 4163236269$

Fax + I 4163232666

Email stephen.halpern@sunnybrook.ca 


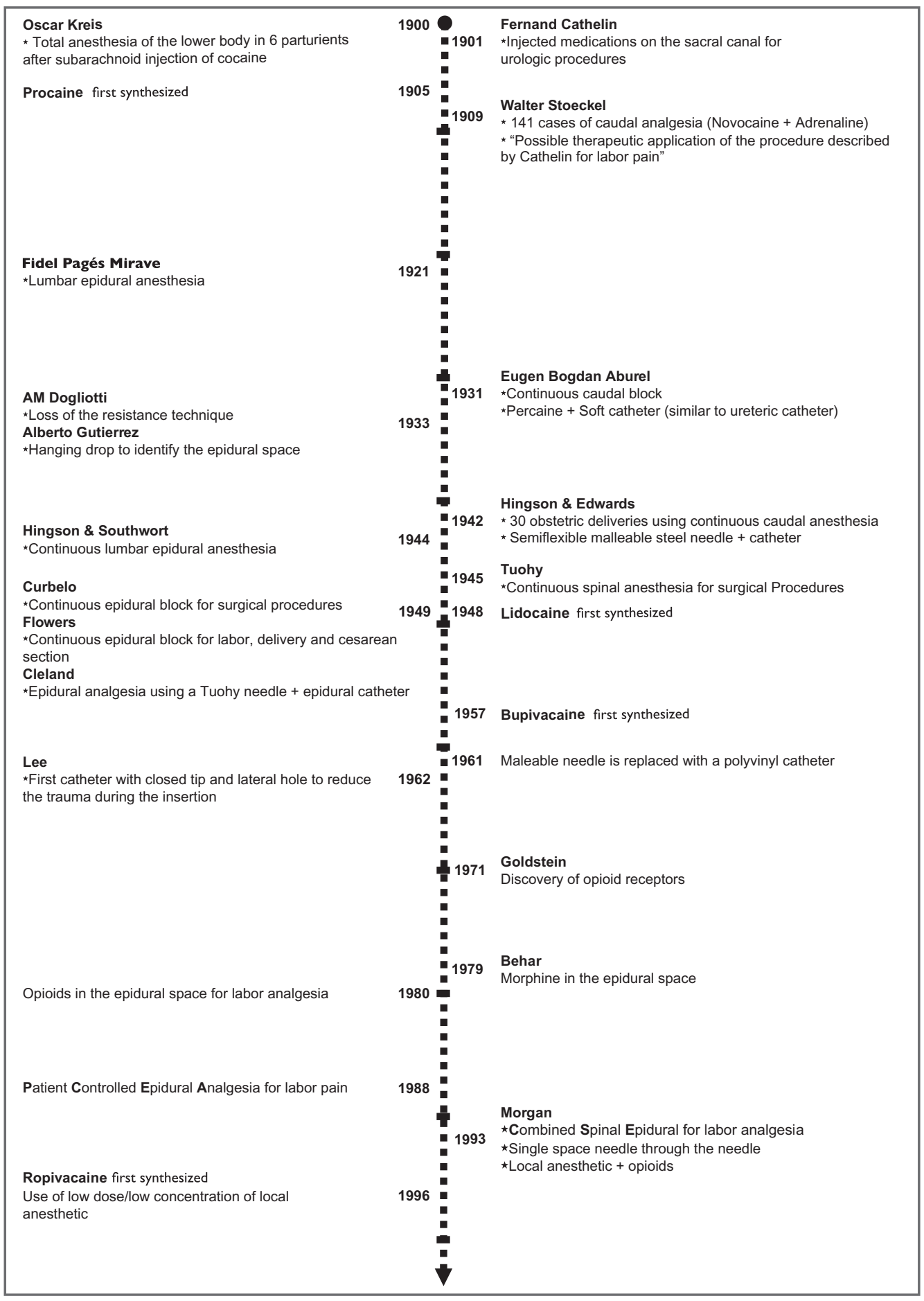

Figure I Timeline illustrating major developments in the use of neuraxial analgesia for labor pain.

In 1909 Walter Stoeckel, a German obstetrician, reported his experience in 141 cases of caudal epidural analgesia for labor pain. He studied healthy parturients of mixed parity. The injections were done at the end of the first stage or during the second stage of labor. His success rate was about $50 \%$, with 16 patients experiencing "very little pain". This technique did not require puncture of the dura mater, and caused severe headache much less frequently than subarachnoid block. He used procaine (novocaine), which had been synthesized in 1905 and was much less toxic than cocaine. This added to the safety of the technique. ${ }^{3}$

The use of a catheter placed into the caudal epidural space was first described by Eugen Bogdan Aburel in 1931. Aburel introduced a needle at the caudal level, then a soft catheter 
was advanced through the needle, after which the needle was removed, leaving the catheter in situ. ${ }^{4}$ This allowed repeated injections throughout labor without the need to repeat the procedure.

Dissatisfaction with neuraxial analgesia occurred because of poor reliability, safety concerns, and the feeling of lower limb paralysis patients experienced when given large doses of local anesthetic. In the early 1960s, the lumbar epidural replaced caudal analgesia as the preferred technique. Compared with the caudal route, lumbar epidural analgesia is more comfortable for the patient and easier to perform. The technique required less local anesthetic. Motor function of the lower extremities and abdominal muscles can be maintained. The extent of sympathectomy can be better controlled, resulting in less maternal hypotension. The block can be extended and used for cesarean section if necessary. During this period of time, bupivacaine was synthesized and became the drug of choice in obstetrics because of its long duration of action and absence of tachyphylaxis. The use of lumbar epidural catheters in the 1970s permitted administration of pain relief early in labor, rather than only at the time of delivery.

Several improvements in epidural analgesia occurred in the 1970s and 1980s. Continuous infusions replaced clinician boluses, leading to enhanced patient safety and satisfaction. ${ }^{5}$ During this period of time, epidural infusion pumps became more compact and reliable. In 1988, Gambling et al described "patient-controlled epidural analgesia" for pain control during labor. ${ }^{6}$ This technique allowed the patient to titrate the amount of drug required to her own needs. Originally, the technique consisted of patient-initiated boluses only, but soon most clinicians included a continuous background infusion in addition to patient-initiated doses. The discovery of opioid receptors in the spinal cord led to the use of opioid/local anesthetic mixtures that further reduced maternal motor block and reduced the risk of local anesthetic toxicity.

More recently, combined spinal-epidural analgesia has become popular. The spinal component provides rapid analgesia with very little motor block of the lower extremities. An epidural catheter is then placed to ensure analgesia is available throughout labor.

\section{Goals of therapy}

Childbirth has been recognized as among the most painful experiences known. Numerous strategies, both pharmacologic and nonpharmacologic, have been used as treatment. However, childbirth is a multidimensional experience and when considering treatment, one must balance between pain relief and other aspects, such as physical, emotional, psychological, sociologic, and sometimes religious considerations. In other words, pain relief may not be enough to make childbirth a fulfilling and satisfactory experience. In this section we will consider the goals of therapy for labor pain and how neuraxial analgesia helps to accomplish these goals.

During the 1930s and 1940s, regional block was rarely used for labor analgesia. Instead, women often received high doses of morphine and scopolamine. This was sometimes supplemented with inhalational analgesia with ether, chloroform, nitrous oxide, or trichloroethylene. These medications were often accompanied by complete loss of consciousness with the accompanying dangers of maternal aspiration and neonatal depression. In addition, amnesia and the inability to participate at the time of delivery resulted in poor patient satisfaction. As a result, better methods of providing pain relief were sought. Table 1 lists some of the characteristics of an "ideal" labor analgesic. The main goal of neuraxial analgesia is to have as many of these characteristics as possible.

\section{Effective pain relief}

Neuraxial analgesia fulfils many of these characteristics. While parenteral opioids may provide sedation, relaxation, and comfort, there is strong evidence to suggest that morphine and meperidine do not decrease pain intensity. ${ }^{7}$ Epidural analgesia provides significantly more analgesia, as measured by visual analog scale in both the first and second stage of labor than parenteral opioid. ${ }^{8}$

\section{Safety}

While side effects can occur, the incidence of permanent maternal injury is low. Neuraxial analgesia results in less neonatal depression than parenteral opioids. ${ }^{8}$

\section{Good progress and outcome of labor}

There have been numerous randomized controlled trials comparing neuraxial analgesia with parenteral opioid. A meta-analysis combined 18 studies comprised of over 6600 patients. $^{9}$ Figure 2 shows the odds ratio (OR) and 95\% confidence interval (CI) for the studies in the meta-analysis.

Table I Characteristics of ideal labor analgesia

- Effective pain relief

- Safe

- Minimal effects on progress or outcome of labor

- Minimal effects on the fetus or newborn

- Minimal maternal side effects

- Lower limb motor block

- Pruritus

- Nausea 


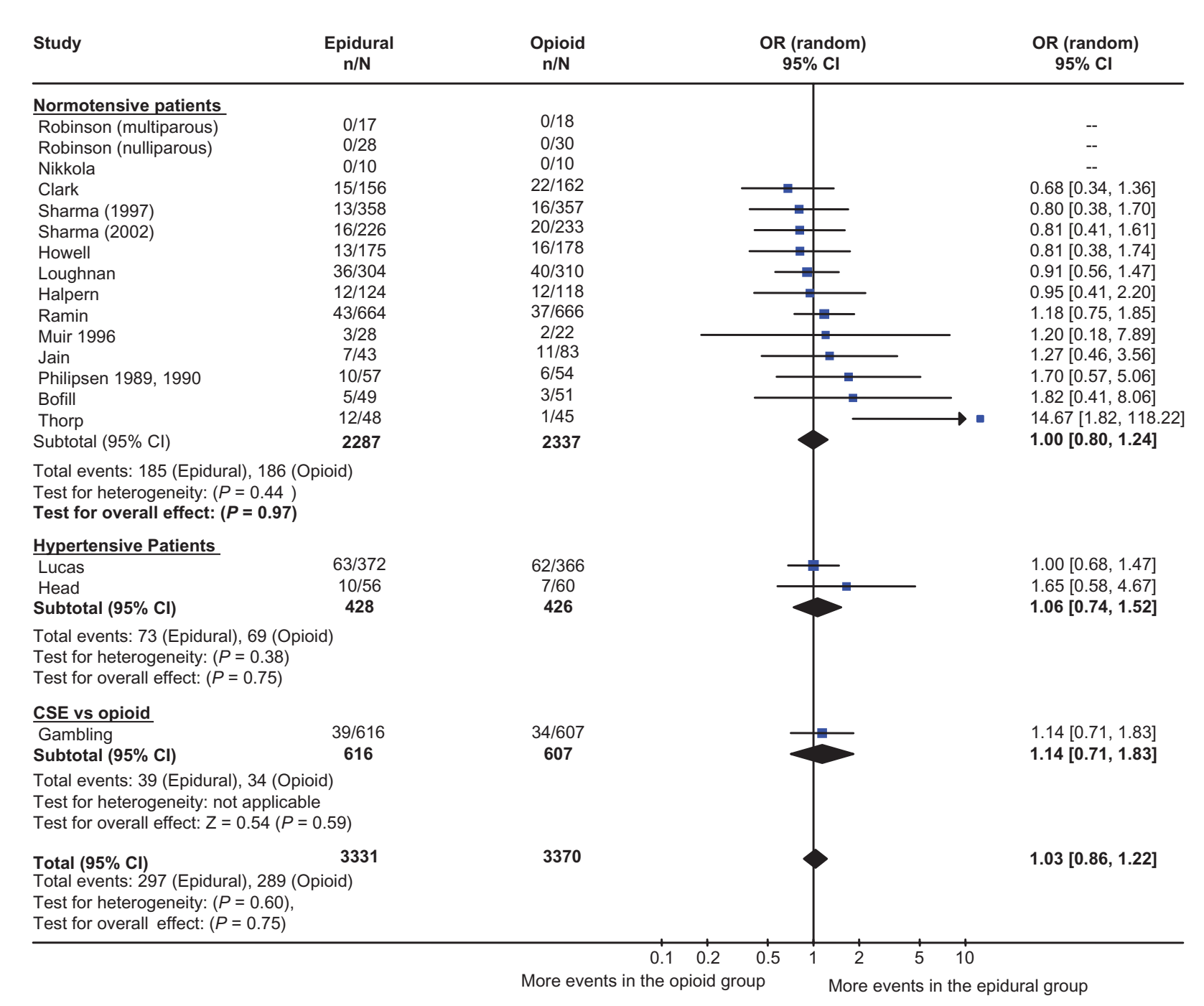

Figure 2 Epidural analgesia versus parenteral opioid analgesia and incidence of cesarean section. The number of patients who had a cesarean section, odds ratio, and $95 \%$ confidence interval are shown for each study. The size of the box is proportional to the weight of the study in the meta-analysis. The scale is logarithmic. For studies with no cesarean sections, the odds ratio could not be calculated. Copyright (C) 2005, Blackwell Publishing. Reproduced with permission from Leighton BL, Halpern SH. Epidural analgesia and the progress of labor. In: Halpern SH, Douglas MJ, editors. Evidence-based Obstetric Anesthesia. Oxford, UK: Blackwell Publishing; 2005.

The incidence of cesarean section was almost identical in the two groups (OR 1.03; 95\% CI: 0.86-1.22). Neuraxial analgesia was associated with a statistically significant increase in the incidence of operative vaginal delivery, but this may have been due to changes in behavior. One of the authors explicitly noted the use of forceps for resident training was facilitated in parturients who received epidural analgesia. ${ }^{10}$ There was no difference in the length of the first stage of labor, although the second stage was a few minutes longer.

\section{Minimal effects on fetus and newborn}

While all medications cross the placenta and may be measured in the newborn, local anesthetics do not cause neonatal depression. Compared with parenteral opioids, neuraxial analgesia is associated with better Apgar scores at one minute and a reduced need for administration of neonatal naloxone. ${ }^{8}$

\section{Minimal maternal side effects}

For the last three decades, research into labor analgesia has been focused on minimizing the maternal side effects of neuraxial analgesia. Several strategies have been explored. These will be discussed in detail in the following sections.

\section{Choice of local anesthetics}

In North America, bupivacaine and ropivacaine are commonly used for labor analgesia. Although there is some experience with levobupivacaine, primarily in the UK, this drug does not seem to have any advantages when compared with the others. Bupivacaine was superior to the older local anesthetics, such as lidocaine, because of its increased duration of action, reduced incidence of tachyphylaxis, and reduced intensity of lower limb motor block. Ropivacaine was synthesized in order to reduce the 
cardiotoxicity associated with bupivacaine and to reduce motor block further. ${ }^{11}$

The use of bupivacaine and ropivacaine in labor has recently been reviewed. ${ }^{12}$ Considering the low doses used for labor, toxicity is rarely associated with either drug. Both are effective analgesics, with little or no difference in maternal satisfaction or effect on labor. There is some evidence to suggest that ropivacaine may produce less motor block in prolonged labors, but the difference may be attributable to differences in drug potency. ${ }^{13}$

\section{Choice of concentration of local anesthetic}

Traditional epidural analgesia was initiated with $0.25 \%$ $0.5 \%$ bupivacaine and maintained with intermittent bolus doses of similar anesthetic solutions. Dense motor block of the lower extremities resulted in dissatisfaction with the technique. Collis et al ${ }^{14}$ conducted a randomized controlled trial that compared bupivacaine $0.25 \%$ to $0.1 \%$ with fentanyl for maintenance of labor analgesia. Using post-partum questionnaires, they found that women who received $0.1 \%$ bupivacaine felt that they had better self-control $(P=0.001)$, less lower limb weakness, and more mobility than the control group. It is also possible that drug concentration may affect mode of delivery. While the cesarean section rate is not affected, COMET (Comparative Obstetric Mobile Epidural Trial) investigators in the UK found an increase in operative vaginal delivery rate in women assigned to maintenance of analgesia using $0.1 \%$ bupivacaine compared with those maintained with $0.25 \% .{ }^{15}$ In both of these trials, there was no difference in the quality of analgesia. These large randomized trials provide sufficient evidence to suggest that low concentrations of local anesthetics provide excellent analgesia and superior maternal satisfaction compared with higher concentrations.

\section{Maintenance of analgesia}

The use of continuous epidural catheters allows maintenance of labor analgesia for prolonged periods of time. Intermittent boluses (by physician or midwife) can provide satisfactory analgesia, but require constant availability of a clinician capable of providing analgesia. Continuous infusions of low concentrations of local anesthetic result in less variability in the quality of analgesia, and require clinician boluses only for breakthrough pain. More recently patient-controlled epidural analgesia has become the preferred technique for maintenance of labor analgesia. This technique has proven to be safe and effective when used with dilute solutions of local anesthetics, with or without a lipid-soluble opioid, such as fentanyl or sufentanil. Clinicians set the bolus dose and lockout interval, and may choose a continuous infusion rate. Compared with continuous infusion alone, patients who receive patient-controlled epidural analgesia require fewer clinician interventions, a reduced dose of local anesthetic, and have less motor block of the lower extremities. ${ }^{16}$ Patient-controlled epidural analgesia superimposed on a continuous infusion further reduces the need for clinician interventions without increasing the incidence of motor block. ${ }^{17}$

There are a wide range of patient-controlled epidural analgesia settings that result in excellent analgesia with minimal motor block. The bolus dose can be set between 4 and $12 \mathrm{~mL}$, with the most common settings between 5 and $8 \mathrm{~mL}$. The lockout interval can be varied, bearing in mind it takes about 10 minutes for the patient to experience pain relief. There is also a wide range of appropriate settings for the background infusion. Low background rates result in more control by the parturient. A recent review discusses these issues in detail. ${ }^{17}$

\section{Combined spinal-epidural analgesia}

The combined spinal-epidural technique results in rapid analgesia with minimal or no impairment of ambulation. Typically, 1 or $2 \mathrm{mg}$ of bupivacaine are combined with 5-15 $\mu \mathrm{g}$ of fentanyl and given intrathecally. An epidural catheter is placed for immediate or later activation using patient-controlled epidural analgesia. This results in profound analgesia that takes effect more quickly than other low-dose neuraxial techniques. There is no difference in obstetric outcomes, such as the incidence of emergency cesarean section or duration of labor. ${ }^{18}$ There is no difference in the incidence of instrumental vaginal delivery when combined spinalepidural analgesia is compared with low concentrations of local anesthetic.

Norris et al enrolled 2183 patients in a study that compared combined spinal-epidural versus epidural analgesia for labor. No difference was found in the incidence of emergency cesarean section or duration of the first or second stages of labor for both techniques. ${ }^{18}$

The COMET study enrolled 1054 patients in three groups, ie, epidural with high-concentration bupivacaine $(0.25 \%)$, epidural with a low concentration of bupivacaine $(0.125 \%$ bupivacaine + fentanyl $2 \mu \mathrm{g} / \mathrm{mL}$ ) and combined spinalepidural analgesia. ${ }^{15,19}$ The investigators found no difference in the incidence of cesarean section, fetal distress, or length of the first or second stages of labor. Patients who received 
combined spinal-epidural and low-dose epidural analgesia had a similar incidence of operative vaginal delivery that was lower than those who received $0.25 \%$ bupivacaine.

An increased incidence of fetal bradycardia may be associated with the use of combined spinal-epidural analgesia. Mardirosoff conducted a systematic review and concluded that the incidence of fetal bradycardia was higher in patients who received a combined spinal-epidural (8.3\% versus $4.7 \%) .{ }^{20}$ However, there was no increase in the rate of cesarean section, operative deliveries, oxytocin usage, or babies with low Apgar score.

The mechanisms through which intrathecal opioids cause bradycardia is not well understood, but it may be related to a rapid decrease in circulating catecholamines, mainly $\beta$-sympathomimetics, secondary to rapid pain relief. The sudden drop in circulating levels of catecholamines, which are known to reduce uterine tone, could increase uterine activity and tone, in turn, can cause a decrease in amount of oxygen delivery to the fetus, leading to fetal bradycardia. An increase in uterine hypertonus has been associated with the use of combined spinal-epidural analgesia. ${ }^{21}$ Therefore, there may be an additional risk of fetal bradycardia, but this does not impact on obstetric or neonatal outcomes.

\section{Side effects and complications}

While neuraxial analgesia is usually safe, complications can occur. Some may be directly attributable to drugs or technique, and these are discussed here and shown in Table 2. Others, such as chronic back pain ${ }^{22}$ and cesarean section, ${ }^{8}$ are attributed to neuraxial block but are not caused by the technique. Finally, the cause of some complications, such as intrapartum fever and breastfeeding difficulties, is unclear.

Table 2 Complications of neuraxial analgesia

\begin{tabular}{|c|c|c|}
\hline $\begin{array}{l}\text { Complication or } \\
\text { side effect }\end{array}$ & Epidural & $\begin{array}{l}\text { Combined } \\
\text { spinal-epidural }\end{array}$ \\
\hline Failure rate 26 & $14 \%$ & $10 \%$ \\
\hline Dural puncture headache & $0.21 \%^{29}$ to $1.6 \%{ }^{18}$ & $0.20 \%^{29}$ to $1.7 \%^{18}$ \\
\hline $\begin{array}{l}\text { Nerve damage cause } \\
\text { by needle trauma }{ }^{35}\end{array}$ & 0.6 per 100,000 & 3.9 per 100,000 \\
\hline Epidural abscess & $\begin{array}{l}0.2-3.7 \text { per } 100.000^{35} \\
3 \text { per } 100.000^{37}\end{array}$ & ? \\
\hline Meningitis & $0-3.5$ per $100.000^{35}$ & $\begin{array}{l}\text { I per } 39,000^{37} \\
0-3.5 \text { per } 100,000^{3}\end{array}$ \\
\hline Epidural hematoma $^{38}$ & 1 in 168,000 & \\
\hline $\begin{array}{l}\text { Fetal heart rate } \\
\text { abnormalities }^{21}(P<0.01)\end{array}$ & $5.5 \%$ & $31.7 \%$ \\
\hline Fetal bradycardia ${ }^{50}$ & $4.7 \%$ & $8.3 \%$ \\
\hline Pruritus $^{20}$ & $29.5 \%$ & $57.8 \%$ \\
\hline
\end{tabular}

\section{Hypotension}

Hypotension is often defined as a $20 \%-30 \%$ drop in systolic blood pressure (compared with baseline) or a systolic blood pressure less than $100 \mathrm{mmHg}$. Because uterine blood flow and fetal oxygenation is directly related to maternal arterial pressure, hypotension is an important side effect that must be treated rapidly. The incidence of hypotension after the initiation of neuraxial analgesia during labor is estimated to be about $10 \%$. This incidence is similar between combined spinal-epidurals and low-concentration epidurals. ${ }^{23}$ The incidence of hypotension is lower in laboring women than in nonlaboring women. Hypotensive episodes are easily treated with complete uterine displacement, additional intravenous fluids, and in some occasions, addition of vasopressors. The treatment should be more aggressive if there is a concerning fetal heart rate pattern or if the mother is symptomatic.

\section{Pruritus}

Pruritus is the most common side effect of neuraxial analgesia. ${ }^{24}$ The incidence and severity is dependent on the opioid dose, and is more frequent with intrathecal opioids than with epidural opioids ( $58 \%$ versus $30 \%$ ). ${ }^{20}$ The cause of pruritus is not well understood, but it is unlikely to be related to histamine release. Antihistamines, often prescribed to treat pruritus after neuraxial opioids, are usually ineffective. There is increasing evidence that neuraxial opioid-induced pruritus is mediated through central $\mu$-opioid receptors. ${ }^{25}$ Opioid antagonists (eg, naloxone) or partial agonist-antagonists (eg, nalbuphine) are effective in relieving pruritus.

\section{Inadequate analgesia}

According to the definition of "neuraxial analgesia failure" employed by researchers, the incidence varies widely among different studies. In a retrospective quality assurance study, Pan et $\mathrm{al}^{26}$ investigated the failure rate with 12,590 neuraxial procedures for labor analgesia in a teaching institution. Failure was defined as epidural or combined spinal-epidural procedures resulting in inadequate analgesia or no sensory block after adequate dosing at any time after initial placement, inadvertent dural puncture by the epidural needle or catheter, intravenous epidural catheter, or any technique requiring replacement or alternative management. The overall failure rate was $12 \%$, being significantly lower after combined spinal-epidural than after epidural analgesia (10\% versus $14 \% ; P<0.001)$. In that study, $5.6 \%$ of the epidural catheters that were initially functioning had to be replaced during the course of labor. Inadequate analgesia with the epidural 
catheter was reported in $8.4 \%$ of the epidural group and in $4.2 \%$ of the combined spinal-epidural group.

\section{Accidental dural puncture and postdural puncture headache}

Accidental dural puncture is an uncommon complication of epidural block. When it occurs, it can produce severe morbidity, although usually of limited duration. The postdural headache that results from accidental dural puncture can severely limit a new mother's ability to care for her newborn. Therefore, these headaches are often treated soon after diagnosis. Epidural blood patch (a sample of the patient's own blood, aseptically drawn and injected into the epidural space) is the most effective treatment of this complication. ${ }^{27}$

Choi et $\mathrm{al}^{28}$ performed a meta-analysis that involved more than 30,000 obstetric patients. They determined that the risk of accidental dural puncture during epidural insertion was $1.5 \%$. Of those patients who had accidental dural puncture, approximately $52 \%$ will result in postdural puncture headache.

van de Velde et $\mathrm{al}^{29}$ reported a retrospective review of more than 17,000 obstetric neuraxial blocks. The overall incidences of accidental dural puncture and postdural puncture headache in this population were $0.32 \%$ and $0.38 \%$, respectively. However if more than one attempt was required to identify the epidural space, the accidental dural puncture rate increased to $0.91 \%$. Fifty-six percent of patients with witnessed accidental dural puncture developed postdural puncture headache. The postdural puncture headache rate was similar between the epidural group $(0.21 \%)$ and the combined spinal-epidural group $(0.20 \%)$. When compared with epidural techniques, the combined spinal-epidural did not protect against accidental dural puncture. The size of spinal needle (27-gauge or 29-gauge pencil-point needle) did not affect the incidence of postdural puncture headache in the combined spinal-epidural group. In this study, $84 \%$ of patients who had postdural puncture headache needed a blood patch, and $15 \%$ needed a second blood patch.

\section{Breastfeeding}

Whether or not neuraxial analgesia may impact breastfeeding initiation and duration is controversial. Observational studies give conflicting results. ${ }^{30,31}$ Beilin et al reported a randomized controlled trial using three different doses of fentanyl for maintenance of epidural analgesia. While there was no effect on breastfeeding initiation, they reported a reduction in breastfeeding frequency at six weeks in the high-dose fentanyl group. ${ }^{32}$ No biologically plausible explanation could be provided. The largest randomized controlled trial on breastfeeding initiation and duration was published by Wilson et al. ${ }^{33}$ In this secondary analysis of the COMET study cited earlier, ${ }^{19} 1054$ nulliparous patients were randomly assigned to a control epidural (bupivacaine, no fentanyl), combined spinal-epidural, or low-dose epidural (both with bupivacaine/fentanyl at initiation and during maintenance). A fourth group comprising matched controls received parenteral pethidine for labor analgesia. There was no difference in the incidence of initiation of breastfeeding among the three groups $(63 \%-66 \%)$. This rate was higher than the pethidine control group (56\%). The duration of breastfeeding was also similar among the three groups (mean 14-15 weeks). This was similar to patients who received parenteral pethidine (14 weeks). The authors concluded that neither epidural analgesia alone or epidural analgesia with fentanyl had any adverse effect on the initiation or duration of breastfeeding.

\section{Nerve damage}

Epidural catheters may injure nerve roots either because they are inappropriately rigid or because they are threaded too deeply and may compress a root, ${ }^{34}$ although a flexible catheter is unlikely to do lasting damage to a nerve root in the epidural space.

In 2009, the Royal College of Anaesthetists in the UK published the third National Audit Project of Major Complications of Central Neuraxial Block. ${ }^{35}$ In total, 320,425 obstetric procedures were analyzed. The incidence of permanent harm after spinal anesthesia was 1.5 in 100,000 , 0.6 in 100,000 after epidural, and 3.9 in 100,000 after a combined spinal-epidural procedure. Overall, in this series, the incidence of permanent harm following neuraxial block was 1.2 in 100,000. Of note, the incidence of nerve damage from obstetric causes unrelated to neuraxial analgesia is almost $1 \%$. $^{36}$

\section{Infection}

Epidural abscess and meningitis are infrequent complications of neuraxial techniques. Reynolds et $\mathrm{al}^{37}$ reported a combination of the findings of 10 surveys. The incidence of epidural abscess after obstetric epidural procedures was $3 / 100,000$. The incidence of meningitis after spinal and combined spinal-epidural anesthesia was 1/39,000. 
Abscess formation complicates $0.2-3.7$ per 100,000 obstetric epidurals. Bacterial meningitis after neuraxial block had a projected incidence of $0-3.5$ in $100,000(95 \% \mathrm{CI})$. It is more frequent after spinal and combined spinal-epidural techniques than after an epidural. ${ }^{35}$

\section{Epidural hematoma}

In spite of the engorgement of epidural veins during pregnancy, epidural hematoma causing neurologic deficits is very rare in the obstetric population, and perhaps the hypercoagulable state of pregnancy acts as a protective factor. In a report of six surveys that together involved more than $1,220,000$ obstetric epidural procedures, one case of epidural hematoma was found. ${ }^{34}$

Ruppen et $\mathrm{a}^{38}$ summarized the results of 27 studies involving 1,370,000 women who had received neuraxial blocks. The risk of epidural hematoma was $1 / 168,000$ overall.

\section{Technique \\ Patient position}

Correct patient positioning is probably the most important factor leading to a successful block. First, it is important to align the vertebrae so that the needle can be inserted between the spinous processes into the epidural space. Twisting of the back will result in the needle contacting the lamina. Second, a position that maximizes the distance between the spinous processes is preferable. Because most parturients at term have a lordotic lumbar spine, maneuvers that flatten or reverse the curvature are advantageous. For example, the "hamstring stretch position" (sitting position with maximum knee extension, hip adduction, and forward lean) has been described to accomplish this. Finally, it is important to be able to identify the midline. In most patients, this can be done by palpating the spinous processes. However, in some patients, the spinous processes are not palpable because of excess adipose tissue or well developed paraspinous muscles. In the sitting position, the midline can be found by drawing a straight line between the vertebra of $\mathrm{C} 7$ (palpable in most patients) and the coccygeal cleft. Ultrasound identification of the midline may be useful to locate the midline, determine the approximate depth to the epidural space, and to determine the level of puncture. ${ }^{39,40}$

Epidural block can be performed in the lateral or sitting position, and the decision is usually based on anesthesiologist and patient preferences. When the spinous processes are not easily palpable, the sitting position is preferred. In patients with easily identifiable landmarks, Vincent and Chestnut ${ }^{41}$ found that neither the lateral nor the sitting position was clearly superior with regard to patient comfort, but heavier patients preferred the sitting position. In some patients, the sitting position may be associated with orthostatic hypotension and syncope. For this reason, it is important for an assistant to provide continuous support to the patient during the procedure. Maternal cardiac output can be reduced in the left lateral position, if held too tightly in position. ${ }^{42}$

\section{Identification of epidural space}

Early methods to find the epidural space relied on identification of negative pressure in the epidural space (eg, hanging drop, Macintosh balloon). The two most common methods used rely on loss of resistance to injection of saline or air as the needle advances through the ligamentum flavum and enters the epidural space. Each technique has its own benefits and drawbacks. Compared with air, loss of resistance to saline has the advantage of providing a more obvious tactile endpoint when the needle enters the epidural space. However, because saline is a clear fluid, it may be confused with cerebrospinal fluid and dural puncture may be masked. A large volume of air or saline should not be injected when confirming needle placement. Large volumes of air may result in inadequate analgesia or patchy block. ${ }^{43}$ Large volumes of saline may result in inadequate analgesia because of dilution. ${ }^{44}$

Schier et a ${ }^{45}$ identified four studies that enrolled obstetric patients who had epidural analgesia and performed a metaanalysis on the results. In these randomized, controlled trials, epidural needle placement was confirmed with loss of resistance to either air or liquid. The outcomes included the incidence of difficulty passing the epidural catheter, intravascular cannulation, paresthesia, dural puncture, postdural puncture headache, and partial block. The authors concluded that the use of air or fluid to identify the epidural space did not change the incidence of any of these outcomes. Recently, a large randomized controlled trial confirmed these results. ${ }^{46}$ This suggests that either method is suitable for epidural placement in labor.

\section{Aseptic technique}

In order to avoid the risk associated with infectious complications of neuraxial analgesia, meticulous aseptic technique should be observed. Because complications such as meningitis and epidural abscess are rare, there are few clinical trials that demonstrate whether or not a particular intervention is useful in preventing infection. Colony counts 
are often reported as surrogate outcomes instead. Recently the American Society of Anesthesiologists published clinical practice guidelines to prevent infectious complications from neuraxial blocks. ${ }^{47}$ While the guidelines recognize limitations in the data, experts in the field and members of the American Society of Anesthesiologists suggest that the precautions listed in Table 3 be employed.

\section{Contraindications}

While neuraxial analgesia is versatile and safe, there are contraindications to the technique. Absolute contraindications include patient refusal, lack of adequate equipment, lack of expertise or supervisory staff, severe coagulopathy, and infection at the site of puncture. Some patients may be technically challenging because of previous back surgery, such as lumbar fusions and Harrington rods. Relative contraindications are listed in Table 4.

Patients with a low platelet count may have a neuraxial block provided they do not have abnormal bleeding. The exact "safe number" of platelets is unknown, but most anesthesiologists would offer neuraxial analgesia if the platelet count was more than $80,000 / \mathrm{mm}^{3}$ and platelet function is normal. Most anesthesiologists consider a platelet count of less than $80,000 / \mathrm{mm}^{3}$ as a relative contraindication to neuraxial anesthesia. ${ }^{47}$

Fever is not a contraindication to neuraxial block. However, if septicemia is suspected, it should be treated with antibiotics before proceeding with neuraxial analgesia. The most common reason for septicemia in the obstetric patient is chorioamnionitis.

Progressive neurologic disease, such as multiple sclerosis, presents a challenge to the anesthesiologist. The disease may progress unpredictably and, if a relapse occurs, neuraxial analgesia may be implicated as a cause. Therefore, it is prudent

Table 3 Recommended aseptic technique for neuraxial analgesia*

Removal of jewelry from hands

Hand washing

Wearing of caps and sterile gloves

Wearing masks that cover both mouth and nose; masks should be changed between cases

Individually packaged skin preparation

Chlorhexidine with alcohol for skin preparation; this must be allowed to dry before needle insertion; povidone-iodine with alcohol is also acceptable

Sterile draping

Sterile occlusive dressing

Note: *American Society of Anesthesiologists Task Force on infectious complication associated with neuraxial techniques. ${ }^{47}$
Table 4 Relative contraindications for neuraxial block

Low platelets but no bleeding diathesis

Infection remote from site of lumbar puncture

Progressive neurologic diseases

Raised intracranial pressure

Hypovolemia

Fixed cardiac output (eg, severe aortic stenosis)

to document any pre-existing neurologic deficits and have a full discussion of the risks and benefits of neuraxial analgesia, preferably before labor starts in order to inform the patient.

Raised intracranial pressure from a supratentorial spaceoccupying lesion is an absolute contraindication to lumbar puncture because the brain may shift down, causing coning and compression of the medulla. In most cases, it is also prudent to avoid epidural analgesia because accidental dural puncture may occur.

Neuraxial analgesia causes a reduction in sympathetic tone, resulting in increased venous pooling in the legs and reduced systemic vascular resistance. This may cause severe hypotension in patients with severe hypovolemia or critical aortic stenosis.

\section{Future directions}

Technologic advances may change the way analgesia is maintained. Recently, Wong et al reported a computer-integrated method that adjusts the background infusion to the number of patient-controlled demands. ${ }^{48}$ Compared with traditional patient-controlled epidural analgesia, this system increased maternal analgesic satisfaction. More recently, intermittent mandatory boluses have been added to patient-controlled epidural analgesia. When compared with a basal infusion, there was a reduction in the amount of local anesthetic used and an increase in patient satisfaction. ${ }^{49}$ Currently, neither of these technologies is available commercially.

\section{Summary}

Neuraxial analgesia is commonly performed to relieve labor pain. Compared with other techniques, it is the most effective form of analgesia. Recent innovations in drug combinations and delivery systems have resulted in a flexible technique that meets the needs of most parturients in a safe and effective manner. The use of low concentrations of local anesthetics, combined with lipid-soluble opioids does not impede the progress of labor or depress the newborn. The addition of patient-controlled epidural analgesia and innovations using new technologies enhance patient satisfaction. 


\section{Disclosure}

The authors report no conflicts of interest in this work.

\section{References}

1. Canadian Institute of Health Information. Highlights of 2008-2009: Selected indicators describing the birthing process in Canada. Available from: http://www.cihi.ca. Accessed 2010 Sep 3.

2. Bucklin BA, Hawkins JL, Anderson JR, Ullrich FA. Obstetric anesthesia workforce survey: Twenty-year update. Anesthesiology. 2005; 103:645-653.

3. Doughty A. Walter Stoeckel (1871-1961): A pioneer of regional analgesia in obstetrics. Anaesthesia. 1990;45:468-471.

4. Curelaru I, Sandu L. Eugen Bogdan Aburel (1899-1975). The pioneer of regional analgesia for pain relief in childbirth. Anaesthesia. 1982;37: 663-669.

5. Evans KR, Carrie LE. Continuous epidural infusion of bupivacaine in labour: A simple method. Anaesthesia. 1979;34:310-315.

6. Gambling DR, Yu P, Cole C, McMorland GH, Palmer L. A comparative study of patient controlled epidural analgesia (PCEA) and continuous infusion epidural analgesia (CIEA) during labour. Can J Anaesth. 1988;35:249-254

7. Olofsson C, Ekblom A, Ekman-Ordeberg G, Hjelm A, Irestedt L. Lack of analgesic effect of systemically administered morphine or pethidine on labour pain. Br J Obstet Gynaecol. 1996;103:968-972.

8. Leighton BL, Halpern SH. The effects of epidural analgesia on labor, maternal, and neonatal outcomes: A systematic review. Am J Obstet Gynecol. 2002;186:S69-S77.

9. Leighton BL, Halpern SH. Epidural analgesia and the progress of labor. In: Halpern SH, Douglas MJ, editors. Evidence-based Obstetric Anesthesia. Oxford, UK: Blackwell Publishing; 2005.

10. Bofill JA, Vincent RD, Ross EL, et al. Nulliparous active labor, epidural analgesia, and cesarean delivery for dystocia. Am J Obstet Gynecol. 1997; 177:1465-1470.

11. Albright GA. Cardiac arrest following regional anesthesia with etidocaine or bupivacaine. Anesthesiology. 1979;51:285-287.

12. Beilin Y, Halpern S. Focused review: Ropivacaine versus bupivacaine for epidural labor analgesia. Anesth Analg. 2010;111:482-487.

13. Halpern SH, Breen TW, Campbell DC, et al. A multicenter, randomized, controlled trial comparing bupivacaine with ropivacaine for labor analgesia. Anesthesiology. 2003;98:1431-1435.

14. Collis RE, Davies DW, Aveling W. Randomised comparison of combined spinal-epidural and standard epidural analgesia in labour. Lancet. 1995;345:1413-1416.

15. Comparative Obstetric Mobile Epidural Trial (COMET) Study Group UK. Effect of low-dose mobile versus traditional epidural techniques on mode of delivery: A randomised controlled trial. Lancet. 2001; 358:19-23.

16. van der Vyver M, Halpern S, Joseph G. Patient-controlled epidural analgesia versus continuous infusion for labour analgesia: A metaanalysis. Br J Anaesth. 2002;89:459-465.

17. Halpern SH, Carvalho B. Patient-controlled epidural analgesia for labor. Anesth Analg. 2009;108:921-928.

18. Norris MC, Fogel ST, Conway-Long C. Combined spinal-epidural versus epidural labor analgesia. Anesthesiology. 2001;95:913-920.

19. Wilson MJ, Cooper G, MacArthur C, Shennan A. Randomized controlled trial comparing traditional with two "mobile" epidural techniques: Anesthetic and analgesic efficacy. Anesthesiology. 2002;97: 1567-1575

20. Mardirosoff C, Dumont L, Boulvain M, Tramer MR. Fetal bradycardia due to intrathecal opioids for labour analgesia: A systematic review. BJOG. 2002;109:274-281.

21. Abrao KC, Francisco RP, Miyadahira S, Cicarelli DD, Zugaib M. Elevation of uterine basal tone and fetal heart rate abnormalities after labor analgesia: A randomized controlled trial. Obstet Gynecol. 2009;113:41-47.
22. Macarthur AJ, Macarthur C, Weeks SK. Is epidural anesthesia in labor associated with chronic low back pain? A prospective cohort study. Anesth Analg. 1997;85:1066-1070.

23. Simmons SW, Cyna AM, Dennis AT, Hughes D. Combined spinalepidural versus epidural analgesia in labour. Cochrane Database Syst Rev. 2007;3:CD003401

24. Herman NL, Choi KC, Affleck PJ, et al. Analgesia, pruritus, and ventilation exhibit a dose-response relationship in parturients receiving intrathecal fentanyl during labor. Anesth Analg. 1999;89:378-383.

25. Ganesh A, Maxwell LG. Pathophysiology and management of opioidinduced pruritus. Drugs. 2007;67:2323-2333.

26. Pan PH, Bogard TD, Owen MD. Incidence and characteristics of failures in obstetric neuraxial analgesia and anesthesia: A retrospective analysis of 19,259 deliveries. Int J Obstet Anesth. 2004;13:227-233.

27. Macarthur A. Postpartum headache. In: Chestnut DH, Polley LS, Tsen LC, Wong CA, editors. Chestnut's Obstetric Anesthesia: Principles and Practice. Philadelphia, PA: Mosby; 2009.

28. Choi PT, Galinski SE, Takeuchi L, Lucas S, Tamayo C, Jadad AR. $\mathrm{PDPH}$ is a common complication of neuraxial blockade in parturients: A meta-analysis of obstetrical studies. Can J Anaesth. 2003;50: 460-469.

29. van de Velde M, Schepers R, Berends N, Vandermeersch E, de Buck F. Ten years of experience with accidental dural puncture and post-dural puncture headache in a tertiary obstetric anaesthesia department. Int $J$ Obstet Anesth. 2008;17:329-335.

30. Torvaldsen S, Roberts CL, Simpson JM, Thompson JF, Ellwood DA. Intrapartum epidural analgesia and breastfeeding: A prospective cohort study. Int Breastfeed J. 2006;1:24.

31. Halpern SH, Levine T, Wilson DB, MacDonell J, Katsiris SE, Leighton BL. Effect of labor analgesia on breastfeeding success. Birth. 1999;26:83-88.

32. Beilin Y, Bodian CA, Weiser J, et al. Effect of labor epidural analgesia with and without fentanyl on infant breast-feeding: A prospective, randomized, double-blind study. Anesthesiology. 2005;103:1211-1217.

33. Wilson MJ, MacArthur C, Cooper GM, Bick D, Moore PA, Shennan A. Epidural analgesia and breastfeeding: A randomised controlled trial of epidural techniques with and without fentanyl and a non-epidural comparison group. Anaesthesia. 2010;65:145-153.

34. Reynolds F. Neurologic complications of pregnancy and neuroaxial anesthesia. In: Chestnut DH, Polley LS, Tsen LC, Wong CA, editors. Chestnut's Obstetric Anesthesia: Principles and Practice. Philadelphia, PA: Mosby; 2009.

35. Cook TM, Counsell D, Wildsmith JA. Major complications of central neuraxial block: Report on the Third National Audit Project of the Royal College of Anaesthetists. Br J Anaesth. 2009;102:179-190.

36. Wong CA, Scavone BM, Dugan S, et al. Incidence of postpartum lumbosacral spine and lower extremity nerve injuries. Obstet Gynecol. 2003;101:279-288.

37. Reynolds F. Neurological infections after neuraxial anesthesia. Anesthesiol Clin. 2008;26:23-52, v.

38. Ruppen W, Derry S, McQuay H, Moore RA. Incidence of epidural hematoma, infection, and neurologic injury in obstetric patients with epidural analgesia/anesthesia. Anesthesiology. 2006;105: 394-399.

39. Balki M, Lee Y, Halpern S, Carvalho JC. Ultrasound imaging of the lumbar spine in the transverse plane: The correlation between estimated and actual depth to the epidural space in obese parturients. Anesth Analg. 2009;108:1876-1881.

40. Arzola C, Davies S, Rofaeel A, Carvalho JC. Ultrasound using the transverse approach to the lumbar spine provides reliable landmarks for labor epidurals. Anesth Analg. 2007;104:1188-1189.

41. Vincent RD, Chestnut DH. Which position is more comfortable for the parturient during identification of the epidural space? Int J Obstet Anesth. 1991;1:9-11.

42. Andrews PJ, Ackerman WE, Juneja MM. Aortocaval compression in the sitting and lateral decubitus positions during extradural catheter placement in the parturient. Can J Anaesth. 1993;40:320-324. 
43. Shenouda PE, Cunningham BJ. Assessing the superiority of saline versus air for use in the epidural loss of resistance technique: A literature review. Reg Anesth Pain Med. 2003;28:48-53.

44. Okutomi T, Hoka S. Epidural saline solution prior to local anaesthetic produces differential nerve block. Can J Anaesth. 1998;45:1091-1093.

45. Schier R, Guerra D, Aguilar J, et al. Epidural space identification: A meta-analysis of complications after air versus liquid as the medium for loss of resistance. Anesth Analg. 2009;109:2012-2021.

46. Grondin LS, Nelson K, Ross V, Aponte O, Lee S, Pan PH. Success of spinal and epidural labor analgesia: Comparison of loss of resistance technique using air versus saline in combined spinal-epidural labor analgesia technique. Anesthesiology. 2009;111:165-172.

47. American Society of Anesthesiologists Task Force on infectious complications associated with neuraxial techniques. Practice advisory for the prevention, diagnosis, and management of infectious complications associated with neuraxial techniques. Anesthesiology. 2010;112:530-545.
48. Wong CA, Ratliff JT, Sullivan JT, Scavone BM, Toledo P, McCarthy RJ. A randomized comparison of programmed intermittent epidural bolus with continuous epidural infusion for labor analgesia. Anesth Analg. 2006;102:904-909.

49. Leo S, Ocampo C, Lim Y, Sia A. A randomized comparison of automated intermittent mandatory boluses with a basal infusion in combination with patient-controlled epidural analgesia for labor and delivery. Int J Obstet Anesth. 2010;19:357-364.

50. Mardirosoff C, Tramer MR. Intrathecal opioids in labor - do they increase the risk of fetal bradycardia. In: Halpern SH, Douglas MJ, editors. Evidence-based Obstetric Anesthesia. Oxford, UK: Blackwell Publishing; 2005.
Local and Regional Anesthesia

\section{Publish your work in this journal}

Local and Regional Anesthesia is an international, peer-reviewed, open access journal publishing on the development, pharmacology, delivery and targeting and clinical use of local and regional anesthetics and analgesics. The journal welcomes submitted papers covering original research, basic science, clinical studies, reviews \& evaluations,

\section{Dovepress}

guidelines, expert opinion and commentary, case reports and extended reports. The manuscript management system is completely online and includes a very quick and fair peer-review system, which is all easy to use. Visit http://www.dovepress.com/testimonials.php to read real quotes from published authors.

Submit your manuscript here: http://www.dovepress.com/local-and-regional-anesthesia-journal 